\title{
SA-DSR: a Bandwidth Optimizing Technic for Dynamic Source Routing Protocol
}

\author{
Sunaina Sharma, Aparna N. Mahajan, Ramesh C. Poonia
}

\begin{abstract}
Anassortment of wireless mobile nodes with no fixed infrastructure is known as Mobile Adhoc NETwork (MANET). MANETs found its application where quick sharing of data is desired without any fixed infrastructure such as online conferences, emergency search during natural disasters management operations, defense, and online meetings and many more. In MANET all the nodes are permitted to change their geographic locations arbitrarily [3]. These networks have dynamic topology which faces the challenge of limited bandwidth wireless network. This is because of the shared nature of MANET which has limited bandwidth, transmission power, limited range of wireless devices and battery limitation. This paper proposed a new protocol namely SA-Dynamic Source Routing Protocol (SA-DSR).
\end{abstract}

Keywords: DSR, SA-DSR, Bandwidth, Battery Saving, Route Discovery, NS-3.

\section{INTRODUCTION}

MANET is self-making, breaking, arranging and regulating network which configures itself without infrastructure whenever it is deployed in a hostile environment. Such kind of topologies changes dynamically and provides challenges of bandwidth management and battery management etc. The routing of the data packets and control packets is in the networkby and large done by inundating. Asthe nodes in MANET grow with the growth of the network,ithas been analyzed that the output for each node deteriorates rapidly[1][6]. The nodes in the linkage continuously move, during that they make and breaka number of links. The routing protocols need to find out the break in the link and reroute the packet to another link. This process consumes a lot of bandwidth and battery [1]. Consequently, bandwidth and battery life are the twokey factors that influence the performance of the MANET. The bandwidthoptimization has been done using the DSR routing protocol which is a Reactive Routing Protocol (RRP) [4]. Here the threshold is applied. The bandwidth is compared with the threshold value. If the value increases the packets drops otherwise continues.

Revised Manuscript Received on February 05, 2020.

* Correspondence Author

Sunaina Sharma, is currently working as an Assistant Professor in Electronics and Communication Engineering at Maharaja Agrasen University.

Dr. Aparna N. Mahajan, Ph.D., Department of Electronics and Communication Engineering Banasthali Vidyapith, Vanasthali, Rajasthan, India.

Dr. Ramesh C. Poonia, Associate Professor of Computer Science at Amity Institute of Information Technology, Amity University Rajasthan India.

(C) The Authors. Published by Blue Eyes Intelligence Engineering and Sciences Publication (BEIESP). This is an open access article under the CC BY-NC-ND license (http://creativecommons.org/licenses/by-nc-nd/4.0/)

\section{I.BASIC MANEUVER}

Existing DSR: The procedure of DSR protocol from the route discovery to route reply has been elaborated in the following procedure:

\section{Routing Procedure of DSR:}

a. Route Discovery: Whenever the node sends data to a destination that is not in direct range it first finds the route using the Route Discovery Contrivance [10].The source node in initializessearches for the route in route cache and if there is no route present in the cache it creates a route request packet (RREQ) [10] and transmits the packet to the network by means of flooding technique [8][9]. Onreceiving the request every neighbor looks up its cache to find an apposite route to this destination if the path is present; it sends the path to the sending node [4]. Else, the neighbor adds its address on the header and rebroadcastthe same route request to there neighbors. Learning from this all the nodes adds information to their cache [3]. The same is accomplished by all the nodes until the destination is reached. If the node has a preexisting trusted route to the destination, the request is then ignored [7][2]. The existing routed can be verified by the unique sequence number of all the nodes. Each node in the network has a unique number which is used to verify that the route is new or existing.

Step:1Initialize the Node ID in the MANET network Initialization source, destination

Step:2 Assign IP and MAC address

Nid->ip_addr,mac_add

For(Source_id,Destination_id)

Step:3 Source_id sends the RREQ message to all neighbor nodes. Source_id wait for RRLP

Step:4 Neighbor nodes find a path to reach Dest at given slot time TX.

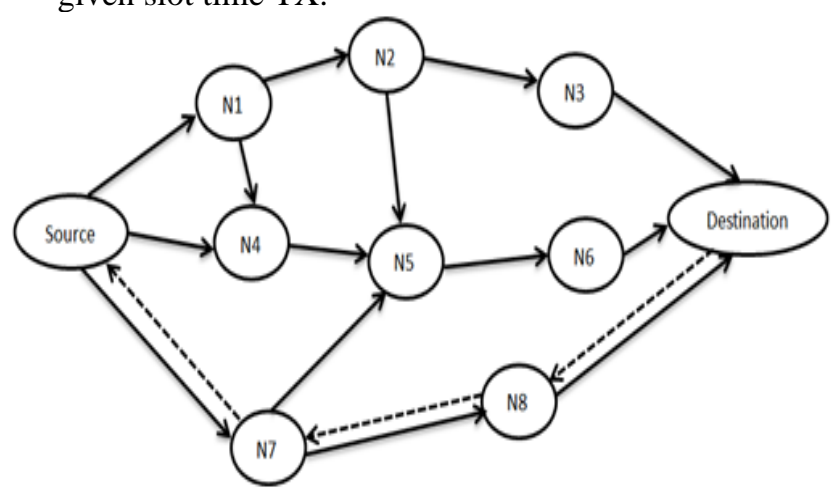

Fig 1: Routing Mechanism of DSR 


\section{SA-DSR: a Bandwidth Optimizing Technic for Dynamic Source Routing Protocol}

Where

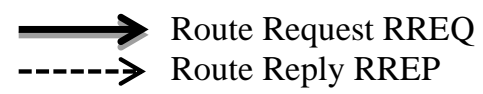

b. Route Reply (RREP): When a node finds the itinerary to a destination it repliesto the sender node with route reply (RREP) message and adds the shortest path from the sender to destination in the cache.

The reply message is a unicast message which has the path in its header as every node adds its unique number into it [5].

Step:5 Maximum number of allowedaccumulation process to be operated at every transmission slots at Transmission seconds.

Step:6 $\mathrm{R}_{\mathrm{x}}=$ Reception Rate

$\mathrm{T}_{\mathrm{r}}=$ Transmission Rate. It may be defined as propagation modification of delay when the transmitterreceiver distance is $\mathrm{Rx}$.

Step:7The receiver node create the shortest path that the middling one-hop neighbor is high.

Step:8 After finding Dest, the Destination node sends RRLP to Source node.

c. Route Error (RERR): Whenever any node fails the sender informs all the other nodes to delete the route containing this node. The information is passed via sending Route Error message (RERR) to all the nodes.

d. Route Maintenance (RM): While sending a packet every midway node checks whether the packet received packet is appropriately received by the next node or not [8]. It also checks if there is some selfish node or dead node in the network. In a dynamic topology where if the node fails tocollect the acknowledgment of the packet within the predefined time of values [9], it resends the packet. Once the limit has been reached it considers the connection as a broken connection and deletes each path containing this connection from its cache [4]. A route error message is then generated and all the intermediate nodes are informed about the failure of the link to delete that route by sending the error message [7].

e. Route Cache (RC): Route cache in routing protocols are used to maintain the recently and commonly used routes. As discovering route evert time may lead to an existingnetwork. It will also use a lot of network resources. As in MANET the nodes have limited resources, it becomes important to conserve the resources for the same reason Route cache is used [7].

Step:10 Source_id sends the packet to the destination node.

Step:11 End

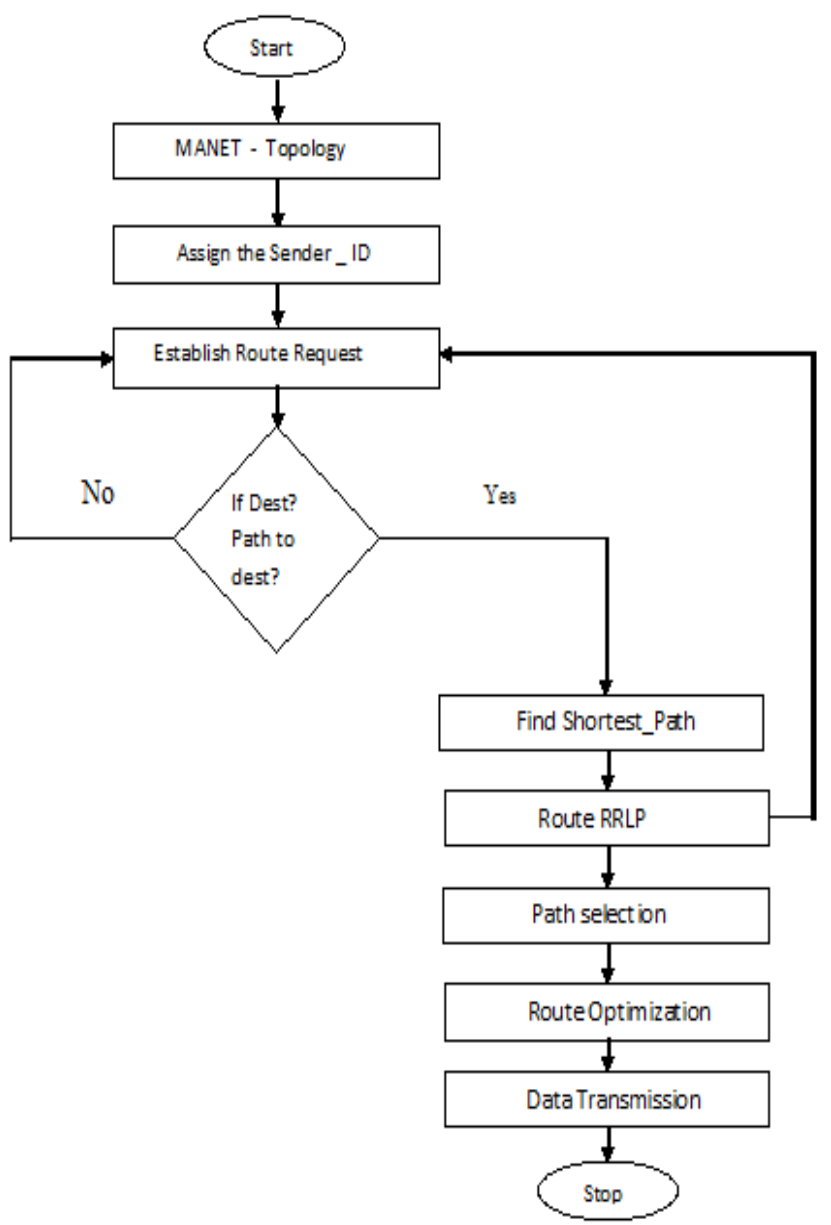

Fig 2: Flow chart of the DSR routing Algorithm

\section{PROPOSED ALGORITHM}

\section{Routing Discovery inSA-Dynamic Source Routing Protocol (SA-DSR):}

a. Route Discovery:As like DSR when a source generates data to the destination that is not in the direct range of destination it will check its cache first. If there is no route to the destination, the source will generate the RREQ packet and flood the neighboring nodes with the RREQ packet until the path to the destination is not confirmed. In SADSR introduces the concept of Threshold. The route discovery process is the same as that of existing DSR with minor changes i.e.it stores the minimum path to the destination in its threshold using the average hop count. For the first time, the minimum route will be the route discovers after that it compares the route and save the shortest route in the threshold. Every time the route is setup it will check whether it is the minimum path to a destination or not. If No, it will again search for a minimum path. If yes, it will check whether there is a scope of further minimization or not. If it further finds any scope it will do the minimization otherwise route the packet. The same can be seen from the flow chart. 
b. Route Reply: When the node discoveries the route from source to the destination it replies to the sender node with Route Reply (RREP) message and adds the shortest path from the sender to destination in the cache which will also include the minimum bandwidth required. The RREP message will contain the unique IDs of the node in its header [5].

c. Route Cache: In SADSR route cache will save the frequently used route with their bandwidthto avoid new route discovery mechanisms. If the route is needed to discover again and again it will require more network resources. So, whenever a new route is requested the SADSR will look into the cache whether they occur or not. If yes, it will check for the bandwidth consumption, if less bandwidth is available then threshold bandwidth it will route the packet to that path otherwise search for another path.

d. Route Error (RERR): Whenever any node fails the sender informs all the other nodes to delete the route containing this node. The information is passed via sending Route Error message (RERR) to all the nodes.

The two factors affecting the network performance are:

Node Constancy (NC): Neighbor node stability shows the reliability of a node in the noise ration to network Signal with a combination of Conventional Signal Strength is a good metric for calculating node stability [11].

Reaming Energy (RE): Energy Spent in transmission and reception mode is a consideration for sending and receiving and receiving packets. Idle mode is not considered here. Congestion Level (CL): It is the ratio of a number of packets in the queue to the buffer size. Queuing Delay (Qdelay): In MANET, processing and transmission delay is constant. Queuing delay is the waiting time in the queue [11].

\section{RESEARCH METHOD}

\section{Algorithm for SA-DSR:}

Step 1: Set the ID of every node in the network.

Step 2: Assign IP and MAC addresses to every node. The node ID will be the composition of node IP address and the MAC address.

$\mathrm{N}_{\text {ID }}=$ ID_address, MAC_Address

Where $\mathrm{N}_{\mathrm{ID}}$ is the node ID

Step 3: After initialing SRC_id and Dest_ID, send a route discovery request from source via flooding all the neighbors with RREQ message. The source will wait for the RRLP message.

Step 4: The neighbors will find the path to the destination within an allotted time period $\mathrm{T}_{\mathrm{x}}$.

Step 5:Find the High BW with the help of value tothe destination

$$
\begin{aligned}
& \text { IF(RSSI }[\mathrm{i}]>=\text { TH[i])then } \\
& \text { Neighbor++,Fid++; }
\end{aligned}
$$

Go to Step 6.

ELSE

\section{Discard the route \\ Go to Step 4.}

Step 6:Here the transmission rate and the reception rate will be the same as that of DSR.

$$
\begin{aligned}
& \mathrm{T}_{\mathrm{r}}=\text { Transmission Rate } \\
& \mathrm{R}_{\mathrm{x}}=\text { Reception Rate }
\end{aligned}
$$

Step 7: After finding Dest with High Bandwidth path, Destination node send RRLP to Source node.

Step 8: SRC_ID accepts the RRLP from Dest_Id.

Step 9: Source_id sends the packet to the destination node through the High Bandwidth Path

\section{FLOW CHART}

The flow chart shows the transmission of data, applyinga top to bottom approach. The complete process can be divided into three parts: Route Discovery (RREQ), Finding high bandwidth and Route Reply (RREP). Fig 3 purposes a new

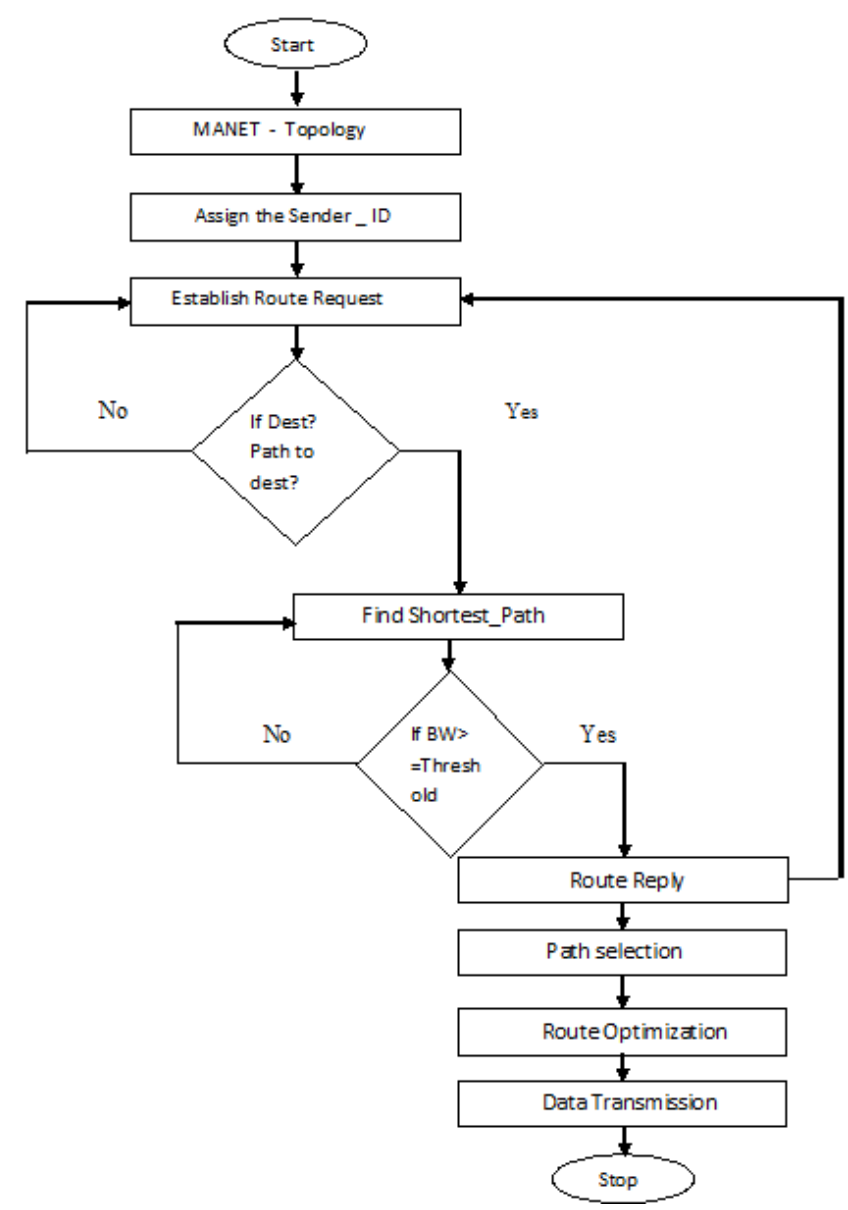

Fig 3: Flowchart for Purposed SA-DSR

Retrieval Number: C5795029320/2020@BEIESP

DOI: 10.35940/ijeat.C5795.029320

Journal Website: www.ijeat.org 


\section{SA-DSR: a Bandwidth Optimizing Technic for Dynamic Source Routing Protocol}

Performance Analysis of SA-DSR: It is observed from the obtainedresult ofresearch work that the average hop count and the end to end delay of the modified DSR i.e. SA-DSR protocol are decreased. As the message goes from one node to other it adds a timestamp and its unique id $\mathrm{N}_{\text {ID }}$ which will consume more bandwidth.

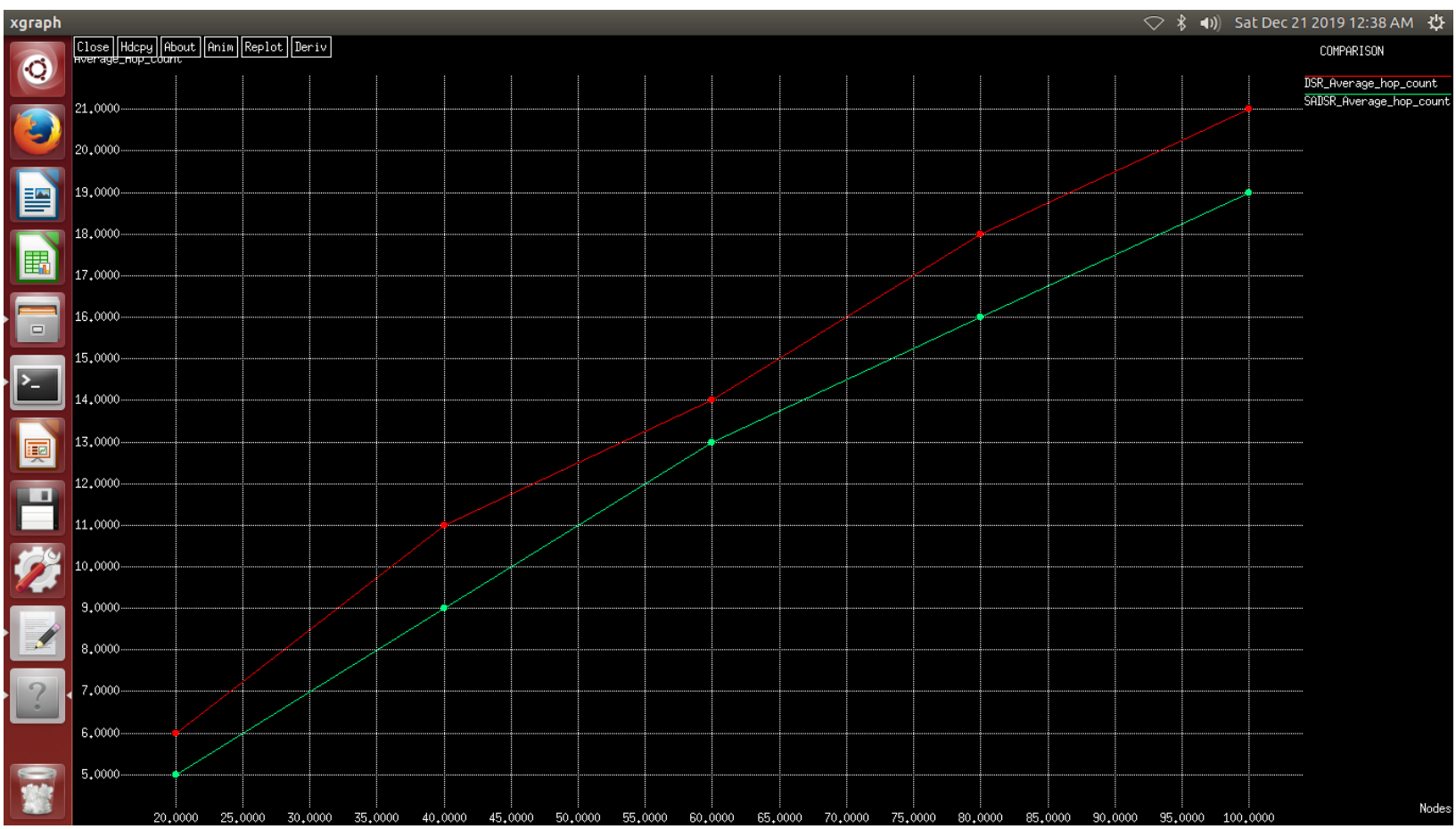

Fig 4: Average Hop Count

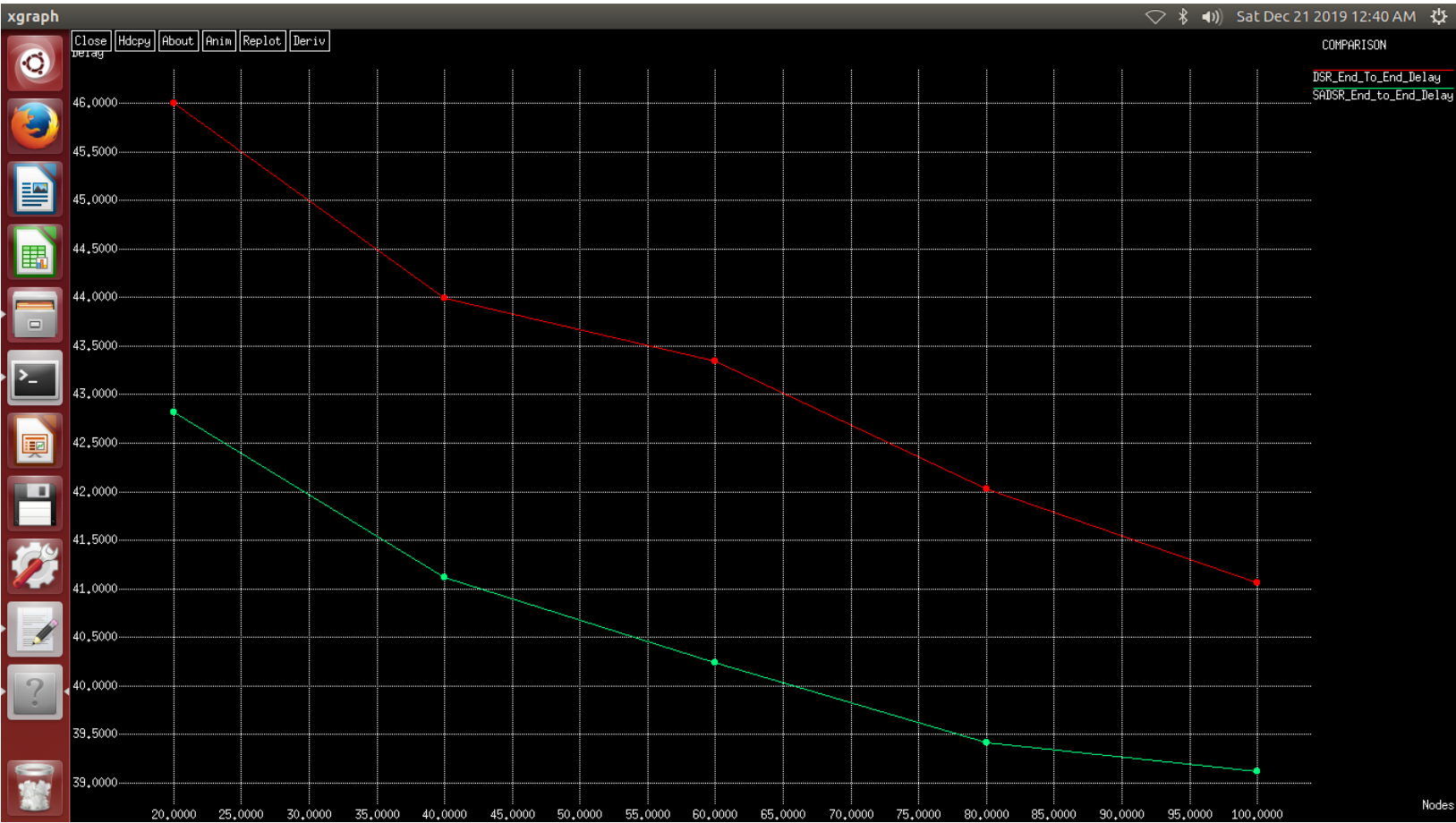

Fig 4: End to End Delay

\section{CONCLUSION}

The paper aimsto optimize the bandwidth in the DSR routing protocol during data communication in MANET. This paper purposes a modified Dynamic Source routing protocol which is Systematic Analysis Dynamic Source Routing protocol (SADSR). The SADSR code has been developed in Network simulator 3.24 as the network simulator provides some inbuilt tools and codes to do so [2]. The research purposes the SADSR with some new concepts for reducing the bandwidth used simultaneously increasing the battery lifetime of the network. Unlike other routing protocols SADSR adapts quickchange in route discovery.A new path selection method in route discovery and route reply have added. The research study shows that theselected path with high bandwidth formula will consumeless bandwidth compared to that of DSR.

\section{Published By:}

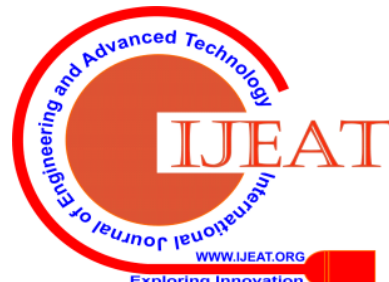




\section{REFERENCES}

1. Heni Kaaniche, Fatma Louati, Mounir Frikha, and Farouk Kamoun, "A QoS Routing Protocol Based On Available Bandwidth Estimation For Wireless Ad Hoc Networks" International Journal of Computer Networks \& Communications (IJCNC) Vol.3 (1), 2011, pp. 219-239.

2. Sunaina Sharma, Aparna N. Mahajan, Ramesh C. Poonia, “An Inclusive survey of Network Simulators", Proceedings of International Conference on Sustainable Computing in Science, Technology and Management (SUSCOM), 2019.

3. Uma Rathore Bhatt, Neelesh Nema, Raksha Upadhyay, "Enhanced DSR: An Efficient Routing Protocol for MANET", International Conference on Issues and Challenges in Intelligent Computing Techniques (ICICT), 2014, pp. 215-219.

4. Bhatia, Bindiya \& Tomar, Parul "Extended Bandwidth Optimized and Energy-Efficient Dynamic Source Routing Protocol in Mobile Ad-hoc Networks" International Journal of Electrical and Computer Engineering (IJECE), 2018. pp. 1460-1466.

5. T. Sudhakarand H. Hannah Inbarani, "Intelligent Path Selection in Wireless Networks using Jaya Optimization", International Conference on Robotics and Smart Manufacturing, (2018) pp. 976983.

6. De-Gan Zhang, Si-Liu, Xiao-Huan Liu, Ting Zhang, and Yu-ya Cui, "Novel Dynamic Source Routing Protocol (Dsr) Based On Genetic Algorithm-Bacterial Foraging Optimization (GA-BFO)", International Journal of Communication System, Dec. 2018.

7. T. Sudhakarand H. Hannah Inbarani, "Intelligent Path Selection in Wireless Networks using JAYA Optimization", International Conference on Robotics and Smart Manufacturing, (2018) pp. 976983.

8. Sunaina Sharma, Aparna N. Mahajan, Ramesh C. Poonia, "A Comparative Study of Modified DSRsRouting Protocols for MANET", International Journal of Innovative Science, Engineering and Technology, Volume 7(2), February 2020.

9. Navin Mani Upadhyay, Kuna Gaurav, and Arun Kumar "Modified DSR, An Energy Conserving Approach to DSR Protocol in MANET", International Conference on Communication and Signal Processing, 2014, pp. 1146-1149

10. Sharmin Sultana, Salma Begum, Nazma Tara, Ahsan Raja Chowdhury, "Enhanced-DSR: A New Approach to ImprovePerformance of DSR Algorithm International Journal of Computer Science and Information Technology, Volume 2, Number 2, 2010, pp. 113-123.

11. Vandna Rani Verma, Dr. D. P. Sharma, and Dr. C.S Lamba, "Stable energy proficient and load balancing based QoS routing in mobile Ad-Hoc networks: Mobile software-based approach", Malaya Journal of Matematik, Vol. 1,2018, pp.79-83.

\section{AUTHORS PROFILE}

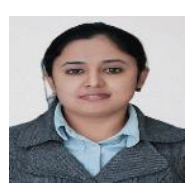

Sunaina Sharma, is currently working as an Assistant Professor in Electronics and Communication Engineering at Maharaja Agrasen University. Her research interest is in the field of wireless (ADHOC NETWORKING) and in security area using Cryptography. She has submitted her Ph.D thesis in wireless and had done her masters from Lovely Professional University. She has eight publications in International journals and presented papers in five conferences.

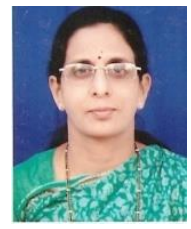

Dr. Aparna N. Mahajan, has done her graduation in Electrical and Power Engineering from Government College of Engineering Amravati, Nagpur University in 1981 and then her post graduation leading to M E in Power System from Govt College of Engineering, Aurangabad. She did her $\mathrm{PhD}$ from Banasthali Vidyapith, Banasthali in the field of Electronics and Communication Engineering in the broad area of Vehicular Adhoc Networking.Dr. Mahajan has over 25 years of teaching and administrative experience and has guided a number of undergraduate and postgraduate projects, with a number of publications in National and International journals and conferences to her credit. Her areas of interest, to name a few, include Computer Networks, Data Communication, Wireless Communication, Mobile Communication etc. She has organized a number of workshops and seminars in these areas as well on Personality development \& communication skills. Dr. Mahajan was past Chairperson of Women in Engineering, IEEE Delhi Section. She was conferred Outstanding Branch Counselor Award from IEEE Delhi Section and IEEE
Head Quarter NewYork in 2015. She was also conferred Best Teacher Award (ECE) in 2006

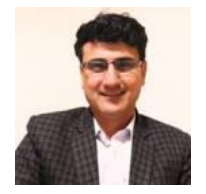

Dr. Ramesh C. Poonia, has rich experience of $15^{+}$ years as an academician. At present he is an Associate Professor of Computer Science at Amity Institute of Information Technology, Amity University Rajasthan India. He has received his PhD in Computer Science from the Banasthali University - Rajasthan - India. His research interests include Internet of Things for Sustainable Agriculture, ITS and WSN Protocol Evaluation, Internet Congestion Control and Simulation \& Modeling. Dr. Poonia has endorsed with the prestigious 'Faculty Appreciation Award' in 2013 for commendable services. He has been engaged as faculty guide for more than 150 undergraduate and graduate students. He has published more than 100 papers and books; some of them have been published in referred journals/conference proceedings as: Elsevier, Springer, Taylor \& Francis, Inderscience IEEE Trans. on Computers, IEEE International Conference on Signal Processing and Communication Engineering Systems, ACM-International Conference Proceedings Series (ICPS). Dr. Poonia is serving as senior member, technical program committee member, member of editorial board/reviewer board of various renowned national and international conferences/journals. 